\title{
L'éducation aux médias dans le Programme de formation de l'école québécoise : intégration, pratiques et problématiques
}

\author{
Normand Landry \\ TELUQ \\ Joëlle Basque \\ HEC Montréal
}

\begin{abstract}
Résumé
Cet article évalue l'état de l'éducation aux médias au Québec. Pour ce faire, il présente et définit d'abord cette notion, pour ensuite en schématiser les ancrages problématiques dans le Programme de formation de l'école québécoise (PFÉQ). Cet article soulève également la question de la formation des enseignants, notamment par une analyse des formations offertes aux professeurs dans les universités québécoises et par la synthèse de quatre entrevues de groupe réalisées auprès d'enseignants de niveaux primaire et secondaire. $\mathrm{La}$ synthèse effectuée permet de problématiser la mise en œuvre des intentions éducatives du PFÉQ en matière d'éducation aux médias à la lumière des perspectives exprimées par des enseignants et des enseignantes. Nos travaux indiquent un soutien minimal offert par le système scolaire québécois se traduisant par la rareté des formations, des ressources et des appuis institutionnels.
\end{abstract}

Mots-clés : conditions de travail, éducation aux médias, formation des enseignants, littératie médiatique, Programme de formation de l'école québécoise

Canadian Journal of Education / Revue canadienne de l'éducation 38:2 (2015)

C2015 Canadian Society for the Study of Education/ Société canadienne pour l'étude de l'éducation 


\begin{abstract}
This article provides an assessment on the state of media education in the province of Québec. It introduces and defines the notion of "media education," and then maps its problematic roots in the Québec Education Program (QEP). The article also raises the issue of teacher training in media education and offers an analysis of current university programs and professional development opportunities available for teachers. Finally, it presents the results of four group interviews conducted with teachers working at primary and secondary levels. The article questions the implementation of the QEP educational aims with regard to media education in the light of perspectives expressed by teachers. It highlights minimal support offered by the school system, resulting in a scarcity of training and resources as well as poor institutional support.

Keywords: media education, media literacy, Québec Education Program, teacher training, work conditions.
\end{abstract}




\section{Introduction}

L'éducation aux médias dispose d'une reconnaissance formelle au Canada. L'inscription de composantes en éducation aux médias dans les curriculums scolaires des provinces et des territoires canadiens a débuté au cours de la seconde moitié des années 1980 pour se compléter au cours de la première moitié des années 2000. Les provinces et les territoires canadiens disposent désormais tous de composantes en éducation aux médias dans leurs curriculums primaires ou secondaires (Andersen, Duncan, \& Pungente, 1999; Pungente, Duncan, \& Andersen, 2005; Landry, Basque, \& Agbobli, 2015). Pourtant, les modalités d'inscription de l'éducation aux médias dans les curriculums scolaires canadiens demeurent peu étudiées (comme exception, on retrouve notamment Wilson et Duncan, 2009). L'influence concrète de cette intégration auprès des pratiques pédagogiques des enseignants et enseignantes est également négligée (sur cette question, voir Dehli, 2009). L'évaluation des objectifs curriculaires en matière d'éducation aux médias dans les provinces et territoires canadiens reste donc problématique.

Cet article s'inscrit dans ce contexte. Il présente en premier lieu une définition de l'éducation aux médias et des concepts qui lui sont associés. Il soulève ensuite les enjeux liés à l'éducation aux médias au Québec et présente les questions de recherche auxquelles cette étude vise à répondre. Une section méthodologique détaille par la suite les travaux d'analyse effectués. Ces travaux procèdent à l'analyse critique et contextualisée de l'insertion de l'éducation aux médias dans le Programme de formation de l'école québécoise (PFÉQ), ceux-ci intégrant à la fois une évaluation de la formation offerte aux enseignants en éducation aux médias et la synthèse des points de vue qu'ils ont exprimés dans le cadre d'entrevues de groupe. Ces éléments précèdent une discussion plus générale sur l'institutionnalisation de l'éducation aux médias en milieu scolaire, ainsi que la conclusion générale. Le présent article offre ainsi des éléments de réponse aux questions suivantes : de quelles manières l'éducation aux médias est-elle introduite dans le Programme de formation de l'école québécoise? Quels sont les thèmes et les objectifs de cette éducation? Quels constats ont été tirés de cette intégration? Quelle est l'influence des dispositions intégrées en éducation aux médias sur les pratiques pédagogiques? 


\section{Éducation aux médias et littératie médiatique : concepts et définitions}

Bien que les fondements de l'éducation aux médias puissent être retracés jusqu'à l'orée du développement des médias électroniques (Fedorov, 2007), celle-ci a réellement pris son envol au cours des années 1980-1990, à la fois comme champ théorique et comme pratique pédagogique. Cette période, notent Hoechsmann et Poyntz (2012), constitue en quelque sorte «l'âge d'or » du mouvement pour une éducation aux médias traditionnelle ayant pour objet les médias de masse, et a connu à la fois une explosion des groupes s'y consacrant et les premières inscriptions formelles de cours d'éducation aux médias dans les cursus scolaires de plusieurs pays, dont le Canada.

En tant que pratique pédagogique, l'éducation aux médias vise cinq grands objectifs : 1) accroître la capacité à employer des technologies médiatiques; 2) développer les compétences permettant de s'exprimer à l'aide de ces technologies; 3) développer des capacités d'analyse critique sur les médias et les textes médiatiques; 4) accroître des capacités d'introspection et; 5) sensibiliser aux enjeux éthiques, moraux et juridiques associés aux médias. Les catégories de savoirs et de compétences recherchées en éducation aux médias sont d'ordre technique, expressif, cognitif, métacognitif et comportemental (pour un survol général de ces questions, voir notamment Martens, 2010; Hobbs, 1998). Ces objectifs généraux se regroupent sous la notion de « littératie médiatique ». Dans sa formulation la plus courante, le concept de « littératie médiatique » se conçoit comme la « capacité d'accéder à des communications, à les analyser, à les évaluer et à en produire dans une variété de formats » (Hobbs, 1996, p. 104). Il s'agit néanmoins d'un terme controversé faisant l'objet de débats récurrents (Lewis \& Jhally, 1998; Hobbs, 1998, 2011; Daley, 2003; Yousman, 2008).

L'éducation aux médias réunit l'usage de dispositifs technologiques, des enseignements sur les médias (considérés ici en tant qu'institutions, acteurs socioéconomiques ou dispositifs technologiques), et un accompagnement introspectif qui interroge l'apprenant sur ses propres valeurs, ses comportements et ses schémas de compréhension de textes médiatiques (Potter, 2013). En d'autres mots, il s'agit de pratiques et de processus éducatifs « destinés à permettre aux membres d'une collectivité de participer de façon créative et critique (au niveau de la production, de la distribution et de la présentation) à l'utilisation des médias technologiques et traditionnels, afin de développer et de libérer les 
individus et la collectivité, et de démocratiser la communication » (cité par Kumar, 1992, p. 167). Toutefois, les innovations technologiques, l'affirmation de nouveaux enjeux associés aux médias, les modifications dans les usages, ainsi que les comportements et la valorisation sociale ou économique de «nouvelles » compétences médiatiques (savoir coder, maîtriser des dispositifs ou des logiciels spécifiques, etc.) obligent à une révision ponctuelle des savoirs et des compétences recherchés en éducation aux médias (Buckingham, 2008; Hartley, 2010). Une ambiguïté sémantique à l'égard de la notion de «média » persiste d'ailleurs et pose certaines difficultés, ce terme évoquant tout à la fois des institutions et des technologies, des outils et des acteurs. Le PFÉQ n'échappe pas à cette ambiguïté.

\section{L'éducation aux médias dans le PFÉQ : une application problématique}

La littérature souligne le caractère problématique de l'application en classe des intentions pédagogiques formulées dans le PFÉQ en éducation aux médias. Le Bilan de l'application du programme de formation de l'école québécoise, dressé par la Table de pilotage du renouveau pédagogique (2006), stipule que « le domaine des Médias est le moins souvent pris en compte par la majorité des enseignants » (p. 103), tant au primaire qu'au secondaire. Il y est également spécifié que c'est « au sujet de la prise de conscience de l'influence des médias dans la société que les plus petites proportions d'enseignants perçoivent de l'amélioration chez les élèves » (p. 103). Selon ce bilan, il y a un consensus au sein des membres de toute l'équipe-école (incluant les membres de la direction et les conseillers pédagogiques), qui s'entendent pour dire que le domaine Médias est le plus difficile à intégrer, le moins abordé et celui où l'on remarque le moins d'améliorations chez les élèves.

Déjà en 1999, Pichette soulignait que « [c]e "nouveau" corpus de connaissances spécifiques sur les médias a du mal à s'arrimer à des disciplines scolaires qui, elles aussi, s'érigent en corpus de savoirs spécialisés avec leur épistémologie, leurs contenus, leurs objectifs didactiques propres et leurs liens privilégiés avec les médias de la culture écrite » (p. 10). Notons également, citant Rossi (2009), qu' « en vertu de la Loi sur l'instruction publique, chaque école est responsable de déterminer à quel(s) moment(s) les professeurs traiteront des médias dans leurs enseignements » (p. 60). Il est donc important 
que le personnel enseignant sache arrimer le domaine général de formation Médias avec les programmes disciplinaires. Nous verrons plus loin que cela n'est pas acquis.

Dans l'avis Soutenir l'appropriation des compétences transversales et des domaines généraux de formation (2007), Marsolais, Desaulniers et Mainville concluent que si l'étude des documents officiels et d'autres systèmes scolaires comme éléments comparatifs confirme la pertinence des domaines généraux de formation et des compétences transversales, elle fait également voir que le soutien et les conseils pour l'intégration de ces composantes sont presque absents du curriculum, livrant ainsi les enseignants à eux-mêmes. Pour le Conseil supérieur de l'éducation, « Si l'État a décidé de traiter les dimensions "santé", "environnement", "médias", "citoyenneté", etc., dans une perspective d'intégration, c'est de son ressort. Cependant, il lui revient aussi d'aider à mettre en valeur cette intégration dans le curriculum et dans les diverses disciplines. En cette matière, [...] le Ministère investit fort peu » (p. 18-19). Cette idée du décalage entre les intentions initiales du curriculum et son applicabilité en classe est reprise par Mellouki (2010) dans Promesses et ratés de la réforme de l'éducation au Québec. Elle suggère qu'il :

existe à la fois une certaine convergence et un certain décalage entre le renouveau pédagogique, tel qu'il est compris et mis en œuvre par les enseignants, et le renouveau tel qu'il est exposé dans les documents officiels. Il y aurait, en d'autres termes, une adéquation sur certains plans et une divergence sur d'autres entre ce qui a été pensé (discours officiel) et ce qui est fait ou faisable, compte tenu des contraintes organisationnelles, matérielles et humaines (discours des enseignants sur leur pratique) (p. 3).

Des questions émergent de ce constat. De quelles manières et dans quelle mesure les objectifs du PFÉQ en regard du domaine général de formation Médias se traduisent-ils en processus pédagogiques concrets ayant lieu dans les disciplines couvertes par les domaines de formation? Quelle formation les enseignants reçoivent-ils au cours de leurs études et au cours de perfectionnement professionnel afin de rejoindre les objectifs du PFÉQ à l'égard du domaine général de formation Médias? Finalement, quelles connaissances, quelles attitudes les enseignants ont-ils à l'égard du domaine général de formation Médias? Nous avons entrepris trois travaux afin de répondre à ces questions : l'analyse systématique de la place occupée par l'éducation aux médias dans le PFÉQ, l'analyse des 
programmes universitaires en éducation au Québec, des formations professionnelles et des structures de support offertes aux enseignants du Québec, et la tenue d'entrevues de groupe réalisées avec des enseignants de niveau primaire et secondaire. Le détail de ces éléments est présenté dans les sections suivantes.

\section{Méthode et analyse}

L'analyse des documents curriculaires devait, en premier lieu, rendre compte de la logique d'intégration suivie par le PFÉQ en matière d'éducation aux médias. La méthode employée a reposé sur l'extraction et l'analyse des sections de texte contenant les occurrences des termes « média(s) », « médiatique(s) » et « médiatisé(e-s) » pour l'ensemble du PFÉQ, tant au primaire qu'aux deux cycles du secondaire. Les sections de texte ayant émergé ont été analysées en fonction de leur emplacement dans les curriculums, des thèmes privilégiés, des intentions pédagogiques qu'elles intègrent et des exercices pédagogiques qui leur sont associés. Cette méthode a permis d'identifier et de détailler les principaux ancrages disciplinaires de l'éducation aux médias dans le PFÉQ. Les inférences indirectes aux médias pouvant être effectuées de la part de thématiques plus générales n'ont pas été retenues. Deux raisons principales expliquent cela : d'une part, l'intégration des inférences indirectes aurait été subjective et dépendante des regards des chercheurs, et d'autre part, l'objectif de la démarche était de systématiser les connaissances sur la distillation des éléments expressément associés au domaine général Médias à travers le PFÉQ.

Dans un second temps, nous avons procédé à l'analyse des programmes de premier cycle pour chacune des universités québécoises offrant des formations conduisant à l'obtention d'un permis d'enseigner. Douze universités québécoises offrent de tels programmes. Cette analyse, effectuée au printemps 2013, a permis d'identifier les programmes qui proposaient des cours se consacrant explicitement, en tout ou en partie, à des questions associées à l'éducation aux médias. L'ensemble des programmes offerts en éducation dans les universités québécoises a donc été analysé afin d'identifier des cours répondant aux critères identifiés (développement de compétences en production et en analyse de contenus médiatiques, enseignement sur les médias considérés comme thème, outils et institutions). L'analyse s'appuie sur les descriptifs (lorsque disponibles) de tous 
les cours des programmes sur les sites Internet des universités québécoises, ainsi que sur l'échange téléphonique avec le personnel administratif des universités. Seuls les cours faisant explicitement mention des critères établis ont été retenus. Cette méthode a ses limites : puisqu'elle porte sur des résumés, donc sur les grandes orientations des cours, elle ne permet pas de fouiller ces derniers en détail mais plutôt de dresser un portrait général de la formation qui affirme contribuer à l'essor des savoirs et des compétences associés à l'éducation aux médias au Québec. Afin de dresser un portrait plus complet de cette formation, nous avons également procédé à une enquête téléphonique auprès des fédérations de syndicats de l'enseignement au Québec, ainsi qu'auprès des syndicats eux-mêmes. Au total, 14 personnes-clés des trois fédérations et des syndicats affiliés ont été interrogées par téléphone en ce qui a trait aux formations offertes aux enseignants dans le cadre de la formation continue. Les entretiens ont été réalisés à partir de questions préalablement élaborées par les chercheurs, et chaque entretien a fait l'objet de notes manuscrites.

Dans un troisième temps, quatre groupes d'enseignants ont été rencontrés : deux groupes d'enseignants du primaire et deux groupes d'enseignants du secondaire. Ces groupes provenaient de la grande région de Montréal. Deux entrevues de groupe ont eu lieu en mai 2013 avec des enseignants du secondaire, ces entrevues réunissant respectivement quatre et trois enseignants. Un directeur s'est joint aux participants de l'une des entrevues pendant son déroulement. Deux entrevues de groupe ont également eu lieu en mai 2013 avec des enseignants du primaire, celles-ci réunissant respectivement trois et six enseignants. Chaque entrevue a duré environ une heure. Des transcriptions textuelles des entrevues ont été rédigées. Les entrevues sont présentées ici à titre exploratoire afin de complémenter l'enquête et l'analyse systématique des curriculums, en ce qui a trait à la formation des enseignants, sous la forme d'une réflexion sur l'intégration de l'éducation aux médias dans les salles de classe. Un questionnaire comprenant cinq catégories de questions a été utilisé. Les enseignants ont répondu aux interrogations, à savoir : 1) quelle était leur attitude par rapport à l'éducation aux médias; 2) s'ils envisageaient de faire de l'éducation aux médias; 3) le cas échéant, quels processus pédagogiques et thématiques ils privilégieraient; 4) à quels obstacles ils se sont butés et quels étaient leurs besoins et; 5) quelle était leur connaissance et leur compréhension des intentions pédagogiques du PFÉQ sur la question. Les transcriptions ont ensuite été analysées dans une perspective double : identification de problématiques précises en regard à la mise en œuvre des 
objectifs du PFQÉ en matière d'éducation aux médias; mise en lumière des perspectives d'enseignants et d'enseignantes sur la question de l'éducation aux médias à l'école. Le résultat de cette démarche ne prétend ni à l'exhaustivité ni à la représentativité. Il se présente comme un coup de sonde visant l'identification de problématiques et d'enjeux vécus par des professionnels dans la mise en œuvre des intentions pédagogiques du PFÉQ liées à l'éducation aux médias.

\section{L'éducation aux médias dans le Programme de formation de l'école québécoise, du préscolaire au secondaire deuxième cycle}

\section{L'éducation aux médias dans la structure générale du PFÉQ}

Trois composantes clés définissent le Programme de formation de l'école québécoise (PFÉQ) aux niveaux primaire et secondaire : les domaines généraux de formation, les compétences transversales et les domaines d'apprentissage. Le Programme comprend « [1] des programmes disciplinaires élaborés à partir d'une approche appelant au développement de compétences; [2] la définition de domaines généraux de formation permettant d'établir des liens entre les apprentissages scolaires et la vie en dehors de l'école; [3] le développement de compétences dites transversales, qui transcendent les programmes disciplinaires » (Table de pilotage du renouveau pédagogique, p. 4). Cette structure vise à la fois l'intégration des enseignements chez les élèves, le développement de compétences jugées nécessaires à leur perfectionnement personnel et leur intégration à la vie sociale et politique.

Tant au primaire qu'au secondaire, les médias constituent un domaine général de formation. Bien que des objectifs précis y soient associés, ce domaine ne fait l'objet d'aucune mesure formelle d'évaluation ou de suivi. L'intégration du domaine général de formation Médias dans le PFÉQ fait suite au rapport Inchauspé (1997), soulignant que « l'omniprésence des médias exige que l'école initie les élèves aux langages propres aux médias, à leur utilisation active, et qu'elle leur fasse acquérir des capacités critiques permettant de résister aux techniques de persuasion » (p. 31).

Deux éléments sont présents tout au long des programmes de niveau primaire et secondaire en éducation aux médias. Premièrement, quatre axes sont ciblés par le domaine général de formation Médias. Ces axes regroupent les thèmes sur lesquels il convient d'interroger les élèves et de développer les activités pédagogiques (MELS, 2006a : éducation préscolaire, enseignement primaire, p. 49). Voici ces axes : 
Tableau 1 : Axes du domaine général de formation Médias

\begin{tabular}{|c|c|c|c|}
\hline $\begin{array}{l}\text { Conscience de } \\
\text { la place et de } \\
\text { l'influence des } \\
\text { médias dans sa } \\
\text { vie quotidienne } \\
\text { et dans la société }\end{array}$ & $\begin{array}{l}\text { Appréciation des } \\
\text { représentations } \\
\text { médiatiques de la } \\
\text { réalité }\end{array}$ & $\begin{array}{l}\text { Appropriation } \\
\text { du matériel et } \\
\text { des codes de } \\
\text { communication } \\
\text { médiatique }\end{array}$ & $\begin{array}{l}\text { Connaissance et } \\
\text { respect des droits } \\
\text { et responsabilités } \\
\text { individuels et } \\
\text { collectifs relatifs } \\
\text { aux médias }\end{array}$ \\
\hline $\begin{array}{l}\text { Fonctions des } \\
\text { médias (informa- } \\
\text { tion, divertisse- } \\
\text { ment, promotion, } \\
\text { influence, propa- } \\
\text { gande); habitudes } \\
\text { de fréquentation } \\
\text { et repères pour la } \\
\text { consommation des } \\
\text { médias; influence } \\
\text { positive ou néga- } \\
\text { tive des messages } \\
\text { médiatiques } \\
\text { sur sa vision du } \\
\text { monde et sur son } \\
\text { environnement } \\
\text { quotidien. }\end{array}$ & $\begin{array}{l}\text { Éléments du lan- } \\
\text { gage médiatique } \\
\text { (son, image, mou- } \\
\text { vement, message); } \\
\text { comparaison entre } \\
\text { les faits et les } \\
\text { opinions; re- } \\
\text { connaissance des } \\
\text { messages sex- } \\
\text { istes, stéréotypés } \\
\text { ou violents; écart } \\
\text { entre la réalité } \\
\text { et sa représenta- } \\
\text { tion virtuelle ou } \\
\text { fictive; qua- } \\
\text { lités esthétiques } \\
\text { des productions } \\
\text { médiatiques; } \\
\text { relations entre des } \\
\text { productions issues } \\
\text { de différentes } \\
\text { techniques et } \\
\text { formes d'expres- } \\
\text { sion esthétiques et } \\
\text { artistiques. }\end{array}$ & $\begin{array}{l}\text { Procédure de } \\
\text { production, de } \\
\text { construction et } \\
\text { de diffusion de } \\
\text { produits média- } \\
\text { tiques; utilisation } \\
\text { de techniques, de } \\
\text { technologies et de } \\
\text { langages divers. }\end{array}$ & $\begin{array}{l}\text { Propriété intel- } \\
\text { lectuelle; liberté } \\
\text { d'expression; } \\
\text { vie privée et } \\
\text { réputation. }\end{array}$ \\
\hline
\end{tabular}

Deuxièmement, la démarche vise à « développer chez l'élève un sens critique et éthique à l'égard des médias et [à] lui donner des occasions de produire des documents médiatiques en respectant les droits individuels et collectifs » (MELS, 2006a : éducation préscolaire, enseignement primaire, p. 340). 
Ainsi, les élèves de niveau secondaire :

seront invités à aborder les productions médiatiques comme des constructions et des « re-présentations » de la réalité qui expriment un point de vue particulier sur des faits, des idées, des valeurs ou des croyances. Ils seront amenés à analyser le message médiatique au regard du contenu, du support technologique, du langage utilisé et du public visé et à en apprécier la valeur esthétique. À travers leurs apprentissages disciplinaires, ils constateront la richesse et la diversité des lectures et des interprétations qu'il est possible de faire à partir des informations véhiculées par les médias. (MELS, 2007 : Programme secondaire $2^{\mathrm{e}}$ cycle : Domaines généraux de formation, p. 11)

Bien qu'elles ne s'y limitent pas, les activités pédagogiques en éducation aux médias offrent une pertinence particulière pour le développement de quatre compétences transversales :

- Compétence 1 : Exploiter l'information;

- Compétence 3 : Exercer le jugement critique;

- Compétence 6 : Exploiter les technologies de l'information et de la communication;

- Compétence 9 : Communiquer de façon appropriée.

Le développement de ces compétences se fait par l'usage de technologies médiatiques ainsi que par la production et l'analyse de contenus médiatiques. À l'instar des domaines généraux de formation, les compétences transversales ne font pas l'objet d'une évaluation formelle par les enseignants. Elles font néanmoins état d'intentions pédagogiques devant guider les enseignants dans l'enseignement des disciplines évaluées. Les ancrages disciplinaires de l'éducation aux médias constituent ainsi un élément d'investigation prioritaire.

\section{Ancrages disciplinaires}

Le Programme de formation de l'école québécoise assure une distillation des thèmes soulevés par les quatre axes du domaine général de formation Médias dans les disciplines formellement enseignées et évaluées. L'éducation aux médias s'effectue conséquemment de manière transdisciplinaire au Québec : au primaire, elle trouve ses principaux ancrages dans les disciplines Arts plastiques, Éducation physique et à la santé et Éthique et culture religieuse; au secondaire, elle s'inscrit principalement en Français, langue 
d'enseignement (seulement pour le $2^{\mathrm{e}}$ cycle), en Anglais, langue seconde et en Arts plastiques. Des processus pédagogiques associés à l'éducation aux médias, tels que l'analyse critique de textes médiatiques et la production de contenus médiatiques, sont toutefois également intégrés dans plusieurs autres disciplines telles que Mathématiques, Science et technologie (et autres disciplines associées), Histoire et éducation à la citoyenneté, Monde contemporain et Éducation physique et à la santé.

Les tableaux qui suivent présentent les principaux ancrages disciplinaires de l'éducation aux médias dans le PFÉQ, du primaire au secondaire. Cela dit, ces tableaux n'ont pas la prétention d'être exhaustifs; des références mineures ont notamment été retirées afin de faciliter la lecture et de cibler les éléments plus significatifs.

Tableau 2 : L'éducation aux médias dans les domaines d'apprentissage : niveau préscolaire et primaire

Domaine d'apprentissage Discipline

Domaine des arts Arts plastiques
Intentions éducatives et thématiques abordées par la discipline

- Cibler son public et communiquer efficacement un message médiatique

- Exploiter des idées de création inspirées par une proposition de création médiatique

- Exploiter des gestes transformateurs et des éléments du langage plastique selon le message et le destinataire

- Organiser les éléments résultant de ses choix selon le message et le destinataire; finaliser sa réalisation médiatique

- Partager son expérience de création médiatique

(MELS, 2006a : éducation préscolaire, enseignement primaire, p. 215)
Domaine du Éducation développement physique et à la personnel santé
- S'adapter aux exigences de la vie contemporaine et porter un regard critique sur les images corporelles véhiculées par les médias

(MELS, 2006a : éducation préscolaire, enseignement primaire, p. 256) 
Domaine d'apprentissage

Domaine de l'univers social

\section{Intentions éducatives et thématiques} abordées par la discipline

- Permettre à ses élèves de s'approprier la réalité d'autres jeunes et de découvrir d'autres espaces culturels en ayant recours aux médias tels que les journaux, la télévision, la radio ou Internet

- Soulever le débat sur les façons de traiter les êtres vivants dans les médias, les rôles et responsabilités de l'homme et de la femme tels qu'ils sont présentés dans les médias, l'esthétique du corps véhiculée dans les médias, l'hypersexualisation des jeunes filles, la discrimination et les préjugés dans son milieu et dans les médias afin d'ouvrir la discussion sur les modes de traitement appropriés et inappropriés ainsi que sur des préjugés, des généralisations et des stéréotypes

- L'analyse médiatique sert de support à une démarche devant amener l'élève à porter un jugement sur la réalité. À partir de la proposition suivante : «Les médias ont de l'influence dans notre société », on propose de :

- Vérifier la provenance des sources : une observation personnelle, un témoignage fiable, une théorie scientifique valable, une autorité reconnue, etc.

- Se questionner sur les raisons qui sous-tendent ce jugement

- S'assurer que la signification du jugement de valeur énoncé est claire

(MELS, 2006a : éducation préscolaire, enseignement primaire, pp. 291, 283, 323, 336, 337) 
Tableau 3 : Secondaire, premier cycle

\section{Domaine d'apprentissage}

Domaine des arts
Discipline

Arts plastiques

\section{Intentions éducatives et thématiques abordées par la discipline}

- Alphabétiser l'élève sur le plan visuel

- Créer des images personnelles, créer des images médiatiques et apprécier des œuvres d'art et des objets culturels du patrimoine artistique, des images personnelles et des images médiatiques

- Maitriser le processus de production médiatique

- Être capable d'identifier des audiences, d'établir des stratégies de communication et de réfléchir sur ces stratégies

- Introspection, réflexion sur son statut de producteur et d'audience

- Analyser des textes médiatiques et des processus de production qui les composent

- Développer des goûts esthétiques et culturels

(MELS, 2006b : enseignement secondaire, premier cycle, pp. 389, 400, 406, 428)

$\begin{array}{ll}\begin{array}{ll}\text { Domaine des } \\ \text { langues }\end{array} & \begin{array}{l}\text { Anglais, langue } \\ \text { seconde }\end{array}\end{array}$

- Explorer des textes oraux, écrits et visuels authentiques

- Réinvestir leur compréhension des textes au fur et à mesure qu'ils explorent, réagissent et produisent différents textes médiatiques

- Réaliser l'ensemble des étapes de production d'un texte médiatique complexe

- Cibler leurs audiences

- Se positionner comme producteurs d'information

- Maîtriser les trois démarches de production médiatique : la préproduction (choisir le texte médiatique à produire, faire une phrase qui résume le projet), la production (créer le texte médiatique en tant que tel) et la postproduction (faire le montage, peaufiner les détails techniques de la production)

(MELS, 2006b : enseignement secondaire, premier cycle, pp. 94, 172, 192, 193) 


\section{Domaine d'apprentissage \\ Domaine des langues \\ Discipline \\ Français, langue \\ d'enseignement}

\section{Intentions éducatives et thématiques abordées par la discipline}

- Comparer les façons de traiter un sujet dans une revue, un film documentaire ou une publicité, par exemple, ainsi qu'à reconnaître le public visé

- Porter attention à la variété de langue utilisée par les personnages, aux clichés, etc.

- Établir ses propres critères graduellement et savoir justifier ses critiques

- Être sensible à ses destinataires et faire des choix en conséquence lorsqu'on produit soi-même des messages

(MELS, 2006b : enseignement secondaire, premier cycle, pp. 94, 97)
- Développer un sens éthique et critique à l'égard des médias par l'exercice du raisonnement mathématique

- Établir des comparaisons et jauger l'écart entre la réalité et l'idée que certains s'en font par la mobilisation de différents modes de représentation, de même que les raisonnements proportionnel, probabiliste et statistique

- Comprendre comment les résultats de sondages constituent des sources d'information dont peuvent s'inspirer les médias pour élaborer articles et éditoriaux

(MELS, 2006b : enseignement secondaire, premier cycle, p. 234)

$\begin{array}{ll}\begin{array}{l}\text { Domaine de } \\ \text { l'univers social }\end{array} & \begin{array}{l}\text { Histoire et } \\ \text { éducation à la } \\ \text { citoyenneté }\end{array}\end{array}$

- Collecter des données provenant de différentes sources médiatiques qui présentent parfois des points de vue opposés ou une information orientée

- Exercer sa pensée critique afin de distinguer entre des faits et des opinions et construire sa représentation des réalités sociales

(MELS, 2006b : enseignement secondaire, premier cycle, p. 340) 
Domaine d'apprentissage

Domaine du développement de la personne

\section{Discipline}

Éducation

physique et à la santé

\section{Intentions éducatives et thématiques abordées par la discipline}

- Se sensibiliser à la violence, au dopage, aux enjeux éthiques, aux messages publicitaires et à l'image corporelle

- Exercer son jugement critique sur ces questions

(MELS, 2006b : enseignement secondaire, premier cycle, p. 472)

Tableau 4 : Secondaire, deuxième cycle

\section{Domaine \\ d'apprentissage Discipline \\ Domaine des \\ langues}

\section{Intentions éducatives et thématiques} abordées par la discipline

- S'informer à partir d'une pluralité de sources (notamment médiatiques)

- Trier, analyser et synthétiser les informations, en identifier les points de vue, être en mesure d'identifier les procédés utilisés dans la construction du texte, être critique et questionner le traitement d'une information médiatique

- Établir le lien entre un média et l'adoption d'un point de vue et comparer ses réactions à l'égard de productions médiatiques avec celles d'autres élèves

- Allier l'exercice du jugement critique sur l'information à la constitution de repères culturels

- Accéder à une lecture de plus en plus distanciée des médias, notamment par la discussion, afin de conduire à l'expression d'un jugement critique éclairé

(MELS, 2007 : Programme secondaire $2^{\mathrm{e}}$ cycle : Français, langue d'enseignement, pp. 3, 14, 29, 33, 90, 101) 


\section{Domaine} d'apprentissage

Domaine des langues

\section{Discipline}

Anglais, langue seconde

\section{Intentions éducatives et thématiques abordées par la discipline}

- Cette discipline reprend plusieurs des objectifs discutés au premier cycle du secondaire

- S'investir dans un processus complet de production médiatique (préproduction, production, postproduction) et analyser les textes médiatiques

- Se faire une opinion sur l'image des adolescents qui est véhiculée par les médias en réinvestissant sa compréhension de différents textes médiatiques (ex. : messages publicitaires, articles de journaux, sites Web)

- Analyser l'information présentée par les médias, en évaluer la justesse, rechercher les stéréotypes, les propos sexistes, les préjugés; discuter de ces éléments avec ses camarades et produire ses propres messages médiatiques adaptés à ses objectifs et publics

(MELS, 2007 : Programme secondaire $2^{\mathrm{e}}$ cycle : Anglais, langue seconde, pp. 15, 56, 57, 58)

Domaine des arts Arts plastiques
- Cette discipline reprend plusieurs des objectifs discutés au premier cycle du secondaire

- Créer des images médiatiques; apprécier des images

(MELS, 2007 : Programme secondaire $2^{\mathrm{e}}$ cycle : Arts plastiques, pp. 19-30) 


\section{Domaine} d'apprentissage

Domaine des mathématiques, de la science et de la technologie

\section{Intentions éducatives et thématiques abordées par la discipline}

- Mobiliser les compétences mathématiques afin de façonner le sens critique, éthique et esthétique à l'égard des médias

- Reconnaître et distinguer les différentes représentations d'objets mathématiques et juger de leur adéquation lorsque sont utilisés du matériel et des codes de communication médiatiques

- S'assurer que les renseignements à caractère mathématique contenus dans les messages sont plausibles

- Mettre à contribution son sens du nombre, son aptitude à analyser des données et ses habiletés de communication pour détecter les intentions de l'émetteur ainsi que les sources de biais qui peuvent influencer son jugement

(MELS, 2007 : Programme secondaire $2^{\mathrm{e}}$ cycle : Mathématiques, p.7) 


\section{Domaine d'apprentissage}

Domaine des mathématiques, de la science et de la technologie

\section{Discipline}

Science et

technologie technologiques et scientifiques

Sciences et technologies de l'environnement

Science et environnement, Chimie

Physique
Applications

\section{Intentions éducatives et thématiques abordées par la discipline}

- S'approprier le matériel et les codes de communication médiatiques

- Constater graduellement l'influence des médias dans sa vie au quotidien et dans la société

- L'enseignant devrait recourir aux différents médias présents dans le quotidien des élèves et les exploiter pour contextualiser les apprentissages et accroître la motivation des élèves sur des questions scientifiques, technologiques et environnementales

(MELS, 2007 : Programme secondaire $2^{\mathrm{e}}$ cycle : Science et technologie, p. 4)

(MELS, 2007 : Programme secondaire $2^{\mathrm{e}}$ cycle : Applications technologiques et scientifiques, p. 5)

(MELS, 2007 : Programme secondaire $2^{\mathrm{e}}$ cycle : Sciences et technologies de l'environnement, p. 5)

(MELS, 2007 : Programme secondaire $2^{\mathrm{e}}$ cycle : Science et environnement, p. 4)

(MELS, 2007 : Programme secondaire $2^{\mathrm{e}}$ cycle : Chimie, p. 4)

(MELS, 2007 : Programme secondaire $2^{\mathrm{e}}$ cycle : Physique, p. 4)

$\begin{array}{ll}\text { Domaine de } & \begin{array}{l}\text { Histoire et } \\ \text { l'univers social }\end{array} \\ & \begin{array}{l}\text { citoyennetion à la } \\ \end{array}\end{array}$

- Questionner les médias comme source et exercice de pouvoir

(MELS, 2007 : Programme secondaire $2^{\mathrm{e}}$ cycle : Histoire et éducation à la citoyenneté, p. 79, 81) 


\begin{tabular}{ll}
$\begin{array}{l}\text { Domaine } \\
\text { d'apprentissage }\end{array}$ & Discipline \\
\hline Domaine de & Monde \\
l'univers social & contemporain
\end{tabular}

\section{Intentions éducatives et thématiques abordées par la discipline}

- S'interroger sur ses propres présupposés (valeurs, convictions, représentations), de même que sur l'influence qu'exercent les médias sur ses vision du monde et sur les sociétés

- S'interroger sur le traitement médiatique d'enjeux contemporains

- Exercer son jugement critique pour discriminer les sources, traiter l'information recueillie et distinguer les faits des opinions

- Développer une pensée critique et un regard éthique à l'égard des médias

(MELS, 2007 : Programme secondaire $2^{\mathrm{e}}$ cycle :

Monde contemporain, pp. 1, 4, 5)

\begin{tabular}{ll}
\hline $\begin{array}{l}\text { Domaine du } \\
\text { développement de }\end{array}$ & $\begin{array}{l}\text { Éducation } \\
\text { physique et à la } \\
\text { la personne }\end{array}$ \\
santé
\end{tabular}

- Poser un regard critique sur des mythes véhiculés par ses pairs et par les médias au regard de la santé

- Questionner les valeurs qui conditionnent les comportements véhiculés par les médias

- Exercer le sens critique sur les messages véhiculés par les médias, notamment en regard à la violence, à l'image corporelle, à l'intégrité, à l'éthique et à la morale dans les activités physiques

(MELS, non daté : Programme secondaire $2^{\mathrm{e}}$ cycle : Éducation physique et à la santé, pp. 4, 5, 23)

\begin{tabular}{ll}
\hline $\begin{array}{l}\text { Domaine du } \\
\text { développement de } \\
\text { la personne }\end{array}$ & $\begin{array}{l}\text { Éthique et } \\
\text { culture religieuse }\end{array}$ \\
\end{tabular}

- Faire preuve de sens critique à l'égard de l'information véhiculée par les médias, par l'examen du traitement médiatique de certaines questions, comme les nouveaux mouvements religieux

(MELS, 2007 : Programme secondaire $2^{\mathrm{e}}$ cycle : Éthique et culture religieuse, pp. 5-6) 


\section{Observations}

Un certain nombre d'observations émergent des tableaux qui précèdent. En premier lieu, la notion de « littératie médiatique », incontournable dans les curriculums scolaires canadiens en matière d'éducation aux médias, est absente du Programme de formation de l'école québécoise. Ce concept occupe pourtant une place centrale dans la littérature scientifique en éducation aux médias (Macedo \& Steinberg, 2007). Le Programme de formation de l'école québécoise fait ainsi l'économie d'un cadre conceptuel à l'intérieur duquel ancrer, regrouper et positionner de manière cohérente les compétences développées par une éducation aux médias. Il s'oppose ainsi au curriculum ontarien, qui intègre formellement la notion de «littératie médiatique » dans les cours de langue anglaise de la

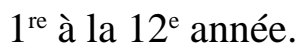

En second lieu, l'éducation aux médias ne constitue pas, au Québec, une discipline à part entière, mais un objet d'enseignement transdisciplinaire devant irriguer les différentes disciplines constituant les domaines d'apprentissage. Ces disciplines devraient à la fois renforcer les processus d'éducation aux médias en intégrant des activités pédagogiques qui s'y rapportent, et mobiliser ces activités afin de renforcer l'acquisition des savoirs disciplinaires enseignés. Cela signifie que la responsabilité des enseignements en matière d'éducation aux médias relève de l'institution scolaire dans son ensemble et que les enseignants spécialisés dans des disciplines particulières devraient être en mesure d'en comprendre les objectifs, les principes et les processus, tout en disposant des compétences pour procéder aux enseignements.

En troisième lieu, le PFÉQ ambitionne de développer la pensée critique sur les médias et les capacités expressives des élèves, tout en contribuant au développement éthique et culturel de ces derniers. Cette approche s'inscrit dans une tradition établie depuis près de trente ans sur la scène nationale et internationale (Masterman, 1985; ministère de l'Éducation de l'Ontario, 1989). Elle évacue néanmoins les enjeux plus récents découlant du repositionnement de la figure du récepteur-consommateur passif ou critique de textes médiatiques (celle-ci étant emblématique à l'ère des médias de masse) vers une figure de producteur et de diffuseur actif de textes médiatiques (Hoechsmann $\&$ Poyntz, 2012). Ces enjeux touchent, entres autres, les pratiques d'intimidation et d'exposition de vie privée, la distribution de matériel diffamatoire et haineux, ainsi que les formes de productions culturelles émergentes reposant sur le recyclage de contenus 
médiatiques. ${ }^{1}$ Ces éléments ne se sont imposés que récemment, et ne faisaient pas l'objet de préoccupations si importantes au moment de l'adoption du PFÉQ.

\section{Formation des enseignants et des enseignantes}

Le développement d'activités pédagogiques en éducation aux médias répondant aux intentions éducatives formulées par le PFÉQ pose comme problématique la question des compétences des enseignants. La présente étude s'est employée à dresser le panorama des offres de formation pour enseignants portant sur cette question précise.

\section{Formation universitaire}

L'ensemble des programmes en éducation conduisant à l'obtention d'un permis d'enseigner a été analysé afin de repérer les cours contribuant au développement des compétences en éducation aux médias. Douze universités québécoises offrent de tels programmes. Les voici :

- Bishop University

- Concordia University

- McGill University

- Université de Montréal

- Université du Québec à Chicoutimi

- Université du Québec à Montréal

- Université du Québec à Rimouski

- Université du Québec en Abitibi-Témiscamingue

- Université du Québec en Outaouais

- Université du Québec à Trois-Rivières

- Université de Sherbrooke

- Université Laval

Trois observations résultent de l'analyse effectuée. En premier lieu, l'intégration de cours associés aux médias dans les formations universitaires des enseignants est

1 Bien que négligées par le domaine général de formation Médias, il n'est pas exclu que ces questions soient traitées ailleurs dans le PFÉQ. Ainsi, la compétence transversale «Exploiter l'information » traite de la question du droit d'auteur. 
presque exclusivement le fait des universités de langue anglaise ${ }^{2}$. À l'exception de la TÉLUQ, qui offre un cours en éducation aux médias, mais qui n'a pas été retenue dans cette liste puisque le cours ne s'inscrit pas dans le cadre d'une formation en enseignement, et de l'UQO, les médias sont absents du programme de formation universitaire. Il est ainsi possible de soutenir que les universités de langue française cumulent un retard par rapport à leurs homologues de langue anglaise. En second lieu, à une exception près, ces cours sont optionnels et ne s'inscrivent pas dans le cursus obligatoire des programmes. Finalement, deux universités seulement, McGill et Concordia, concentrent la quasi-totalité des cours touchant aux médias. Cela signifie que les étudiants qui évoluent dans dix des douze universités offrant des programmes en éducation n'ont qu'un accès limité à ces cours dans le cadre de leur parcours académique. De plus, les programmes en éducation ne permettent généralement pas à l'étudiant de suivre des cours optionnels libres. Cependant, chaque programme contient un ou plusieurs cours où l'étudiant prend connaissance du programme du Ministère qui est propre à sa discipline d'enseignement. Puisque l'éducation aux médias fait partie des programmes de formation du ministère de l'Éducation, du Loisir et du Sport, il est possible de penser que ces cours, nommés didactique dans les programmes universitaires (ex. : didactique des sciences, didactique du français, etc.), abordent des aspects de l'éducation aux médias. Une même possibilité prévaut pour les cours s'intéressant à l'intégration des TIC dans l'enseignement; il est concevable que ces derniers abordent des questions, des thèmes et des compétences associés à l'éducation aux médias, bien qu'ils n'en fassent pas explicitement mention dans leurs descriptifs généraux.

Au final, le panorama est le suivant : concentration des cours qui soutiennent touchent à l'éducation aux médias dans deux universités québécoises de langue anglaise; programmes en éducation offrant peu ou pas de latitude pour des cours optionnels libres; possibilité non vérifiée que des aspects touchant à l'éducation aux médias soient abordés dans des cours visant l'enseignement des disciplines spécifiques ou l'usage de technologies médiatiques à des fins pédagogiques.

2 Ces cours sont les suivants : McGill : EDEC 612 Media Literacy; DPT 420 Media Literacy for Education; COMS 300 Media and Modernity in the 20th Century; COMS 210 Introduction to Communication Studies; COMS 330 Media in Cultural Life; COMS 200, History of Communication. Concordia : EDUC 411, Toys, Media Literacy and Children's Popular Culture; EDUC 405 Children and Technology. UQO : ARI1043 Image et société. 


\section{Formation continue}

Il n'existe aucun programme de formation continue ou de perfectionnement professionnel en éducation aux médias offert par les trois principales fédérations de syndicats de l'enseignement au Québec, nommément la Fédération des syndicats de l'enseignement du Québec (FSE), la Fédération autonome de l'enseignement (FAE), ainsi que la Fédération nationale des enseignants et enseignantes du Québec (FNEEQ). Le portrait qui se dégage des informations recueillies auprès des fédérations ainsi qu'auprès de certains de leurs syndicats membres indique très clairement qu'aucun effort concerté de la part des organisations syndicales n'existe en ce moment afin d'outiller les enseignants de niveau primaire, secondaire et collégial à dispenser une formation sur les médias. Cela n'exclut pas la possibilité que des regroupements syndicaux locaux puissent offrir de tels services. Il en résulte toutefois l'absence d'une formation uniforme et commune aux membres de chacune des trois principales fédérations de syndicats d'enseignement du Québec.

Les travaux de recherche effectués ont néanmoins permis de positionner le regroupement de conseillers pédagogiques RÉCIT comme le meilleur point d'ancrage pour le développement des compétences en éducation aux médias chez le corps enseignant. Le RÉCIT est un réseau de personnes-ressources mis au service du personnel enseignant des différentes commissions scolaires ${ }^{3}$. Son mandat est de favoriser l'intégration pédagogique des technologies de l'information et de la communication (TIC) en milieu scolaire; il s'oriente principalement dans une optique d'éducation par les médias. Cela dit, il est apparent que le personnel du RÉCIT intègre des dimensions critiques aux médias dans le cadre de ses activités. Des formations et des outils traitant des enjeux éthiques aux médias sociaux sont notamment offerts. Le RÉCIT se présente ainsi comme un acteur important sur la question de l'éducation aux médias.

3 Pour un survol des activités menées et des thématiques abordées, voir le site Web du RÉCIT à cette adresse : http://recit.qc.ca/ 


\section{Pratiques pédagogiques en milieu scolaire}

L'étude des perspectives exprimées par les enseignants eux-mêmes sur l'éducation aux médias semble être une nécessité afin de comprendre les difficultés qui y sont associées.

La synthèse des transcriptions des entrevues réalisées auprès de quatre groupes d'enseignants permet d'identifier les points suivants en tant que principaux éléments soulevés au cours des discussions.

Tableau 5 : Entrevues

\section{Synthèse}

Les enseignants sont généralement disposés à développer des activités en éducation aux médias et disposent d'une grande liberté académique leur permettant de moduler leur cours et d'intégrer les éléments qu'ils jugent pertinents et importants
La plupart des enseignants considère importants les thématiques et enjeux soulevés en éducation aux médias

\section{Extraits d'entrevue ${ }^{4}$}

On en fait nous parce qu'on est intéressés par les médias, mais y'a rien, ou presque, dans le cours d'éthique comme tel, qui vient parler des médias. Il peut y avoir un exercice ou un atelier, mais il n'y a pas quelque chose dans le programme. Nous on utilise les médias, puis on les intègre au programme. (Roger, Éthique et culture religieuse, secondaire)

4 Les noms des personnes interviewées ont été modifiés afin de préserver leur anonymat. 
Synthèse

Il existe une méconnaissance significative chez les enseignants des objectifs, des thèmes et des ancrages de l'éducation aux médias dans le PFÉQ

\section{Extraits d'entrevue ${ }^{4}$}

Donc c'est ça, une fois qu'ils ont trouvé quelques articles de journaux sur leur sujet ben là ils les résument puis ils prennent position. Est-ce que c'est de l'éducation aux médias? Encore une fois, peut-être indirectement, parce qu'ils voient bien que les journalistes ne traitent pas un sujet de la même façon, parce que par exemple tel journal va avoir tel parti pris, tel autre journal, un autre parti pris. Par exemple, les journaux occidentaux vont avoir une teinte, alors que d'autres journaux vont avoir une autre position. Donc, ils sont capables de le faire, mais ça ne fait pas partie de notre programme. On le fait parce que c'est tellement présent aujourd'hui, on ne peut pas faire abstraction de ça, mais ça ne fait pas partie du programme. Je ne me sens pas obligée de le faire en fait. (Sara, Français, $5^{\mathrm{e}}$ secondaire)

Les enseignants rapportent qu'il n'existe, à leur connaissance, aucun programme de formation continue ou de perfectionnement professionnel en éducation aux médias au Québec

Le positionnement des médias en tant que domaine général de formation ne favorise pas sa considération par les enseignants, les directions d'école et les commissions scolaires en tant que priorité
Peut-être si on avait une formation quelconque qui touche notre niveau, si quelqu' un venait nous former pour nous donner des trucs pour attirer encore plus leur attention, pour savoir comment aborder telle nouvelle plus délicate, peut-être que ça nuirait pas. (Audrey, primaire, $4^{\mathrm{e}}$ année)
C'est un aspect qui est dans les programmes de formation, mais je pense que ça passe inaperçu [l'éducation aux médias]. C'est vraiment dans la volonté de l'enseignant de l'intégrer, mais il n'y a pas de vérifications, il n'y a pas d'exigences non plus. C'est pour ça que nous on veut prioriser peut-être plus les formations reliées à la pédagogie de leurs matières, que d'essayer d'intégrer un autre sujet comme les médias. Déjà c'est complexe ce qu'ils ont à enseigner et leurs tâches, donc leur donner une autre responsabilité... (Ned, directeur d'une école secondaire) 
Synthèse

Le corps enseignant se considère en surcharge et résiste à une tâche éducative supplémentaire;

\section{Extraits d'entrevue ${ }^{4}$}

Ben moi en fait, c'est super intéressant, les médias et tout, mais c'est parce que à un moment donné notre tâche s'alourdit vraiment beaucoup. Ils veulent tout le temps nous en ajouter beaucoup, encore plus. Je comprends que c'est important d'enseigner aux jeunes (les médias), mais je pense qu'à un moment donné va falloir voir c'est quoi l'essentiel, parce que à quelque part l'éducation part aussi des parents. Ils ont une part de responsabilité à faire. Puis moi j'ai l'impression, je sais que je suis en début de carrière, mais je trouve qu'il y a beaucoup de choses qui sont mises sur nos épaules, y'a beaucoup de choses que c'est nous qui faisons l'éducation aux enfants, aux élèves, mais y'a des choses qui devraient être vues du côté des parents. (Sylvie, primaire, $5^{\mathrm{e}}$ et $6^{\mathrm{e}}$ année)

\section{Problèmes concrets soulevés par les enseignants}

Les principaux obstacles à l'intégration plus poussée de l'éducation aux médias dans l'école sont les suivants : la lourdeur de la tâche, le manque de temps, la priorisation relativement basse du sujet, la responsabilité partagée avec les parents en la matière : l'absence de formation. En somme, les enjeux touchant la formation, les conditions de pratique et les priorités scolaires sont d'une importance considérable dans la volonté et la capacité des enseignants rencontrés de réaliser les objectifs du PFÉQ en matière d'éducation aux médias. L'éducation aux médias s'aborde ainsi sous le prisme des conditions de travail du corps enseignant. Elle doit tenir compte des besoins des enseignants, des ressources auxquelles ils ont accès et de leur charge de travail (Scheibe \& Rogow, 2012). 


\section{Discussion des résultats}

Les enjeux soulevés par notre recherche s'inscrivent dans le cadre d'une institutionnalisation progressive de l'éducation aux médias dans les systèmes scolaires canadiens et étrangers. Cette institutionnalisation devait atteindre deux objectifs étroitement associés : l'étalement progressif des enseignements associés à l'éducation aux médias (sur l'ensemble des institutions d'enseignement primaire et secondaire) et l'inscription formelle de l'éducation aux médias dans les programmes de formation (Frau-Meigs \& Torrent, 2009). Ces objectifs sont ceux d'une généralisation et d'une institutionnalisation de l'éducation aux médias en milieu scolaire. Des progrès appréciables ont été réalisés sur ces points. Il existe désormais des réseaux internationaux composés de chercheurs, d'éducateurs, de parents, de fonctionnaires, de représentants élus, de centres de recherche ainsi que d'institutions nationales, régionales et internationales qui se consacrent à l'éducation aux médias. Ces réseaux s'emploient à contribuer à l'élaboration de politiques et d'initiatives favorables à l'éducation aux médias, à la sensibilisation, à l'éducation de publics cibles, à la formation des éducateurs et au développement d'outils pédagogiques.

Tant à l'international qu'à l'intérieur des pays qui s'y investissent, il est possible de synthétiser le développement de l'éducation aux médias en une démarche commune établie en cinq étapes :

1. L'expérimentation : développement de démarches préliminaires d'éducation aux médias par des précurseurs, essentiellement en milieu communautaire ou scolaire

2. Le regroupement : établissement d'organisations visant à définir le cadre général, le contenu et les meilleures pratiques de l'éducation aux médias, et d'en étendre la pratique

3. La mobilisation : représentation auprès des autorités scolaires, administratives et politiques afin de formaliser l'éducation aux médias par une intégration plus ou moins formelle dans des cursus scolaires et l'obtention de ressources plus conséquentes

4. L'institutionnalisation : l'intégration de pratiques d'éducation aux médias dans le cadre de curriculums scolaires ou par des activités communautaires financées par des partenariats 
5. Le support institutionnel : l'investissement de ressources en formation, en outils et en temps de travail nécessaire à l'atteinte des objectifs établis à l'étape précédente (pour une perspective historique, voir : Fedorov, 2007; Piette, 1996; Masterman, 1985)

Chacune de ces étapes est associée à divers niveaux de pérennité, de formalisation et de reconnaissance de l'éducation aux médias. Les problématiques soulevées dans le cadre de notre recherche tendent à inscrire et à cantonner le Québec à la quatrième étape. L'absence d'un support institutionnel appréciable rend improbable l'atteinte des intentions éducatives par le PFÉQ en matière d'éducation aux médias et mine, à terme, la légitimité de l'institutionnalisation de l'éducation aux médias au sein du système scolaire québécois. Sur cette question précise, la formation des enseignants à l'éducation aux médias apparaitt comme un enjeu incontournable qui pose des difficultés particulières. Les technologies, les priorités, les enjeux et les thèmes associés à l'éducation aux médias évoluent rapidement (Jenkins et al., 2009; Livingstone, 2004), ce qui pose comme exigence une actualisation continue des compétences et des savoirs des enseignants. Qui plus est, l'éducation aux médias demande des investissements récurrents en ressources matérielles, humaines et financières. Or, le support institutionnel minimal offert par le système scolaire québécois en matière d'éducation aux médias, qui se traduit par l'absence d'une formation adéquate et une pénurie de ressources, contredit cette exigence. Cette situation est d'autant plus paradoxale que les enjeux traités en éducation aux médias occupent désormais un espace public et politique appréciable et font l'objet de préoccupations parentales et scolaires accrues.

\section{Conclusion}

Notre étude aura permis de définir la logique d'intégration de l'éducation aux médias dans le PFÉQ, d'en cibler les principaux contenus et points d'ancrage, puis d'en problématiser les intentions éducatives à la lumière des perspectives exprimées par les enseignants. Rossi (2009) soulève une question fondamentale en soutenant que : « si la pertinence d'une éducation aux médias est désormais reconnue, l'évaluation de cette éducation s'avère donc tout aussi importante. Or les données évaluatives de l'éducation aux médias et de sa mise en place demeurent à ce jour relativement dispersées et 
fragmentaires » (p. 50). Tant l'application des intentions éducatives en éducation aux médias que l'état des connaissances sur les processus pédagogiques déployés en classe demeurent insatisfaisants. Des travaux de recherche subséquents devraient approfondir le rapport entretenu entre le PFÉQ et les pratiques pédagogiques déployées en classe, notamment à l'égard des inférences indirectes aux médias, que l'on retrouve dans les textes qu'il regroupe, et aux thèmes généraux qu'il met de l'avant et qui pourraient servir de levier à des enseignements sur les médias. Ces recherches devraient également identifier plus précisément les besoins des enseignants et enseignantes en terme de support institutionnel. La systématisation des connaissances sur le rapport entretenu entre littérature scientifique, curriculum scolaire et pratiques pédagogiques en éducation aux médias au Québec se présente désormais comme une condition de sa mise en œuvre effective et la réalisation des intentions pédagogiques qui la guident.

\section{Remerciements}

Les auteurs tiennent à remercier Élisabeth Routhier et Julie Daubois pour leur importante contribution aux travaux de recherche. Les auteurs remercient également le Fonds de recherche sur la société et la culture, pour son soutien financier et le Conseil de presse du Québec, pour lequel un rapport d'expertise a été rédigé.

\section{Références}

Andersen, N., Duncan, B., \& Pungente, J. (1999). Media education in Canada - The second spring. Dans C. von Feilitzen \& U. Carisson (éds), Children and media: Image, education, participation. Children and media violence. Yearbook from the UNESCO international clearinghouse on children and violence on the screen (pp.139-162). Göteborg, Suède : UNESCO, Nordicom.

Buckingham, D. (2008). Defining digital literacy - What do young people need to know about digital media? Dans C. Lankshear \& M. Knobel (éds), Digital literacies: Concepts, policies and practices (pp.73-91). Oxford, Angleterre : Peter Lang.

Daley, E. (2003). Expanding the concept of literacy. Educause Review, 38(2), 32-40. 
Dehli, K. (2009). Media literacy and neo-liberal government: Pedagogies of freedom and constraint. Pedagogy, Culture \& Society, 17(1), 57-73.

Fedorov, A. (2007). Media education: A historical perspective.

Nordicom. Repéré à : http://www.mediagram.ru/netcat_files

/108/110/h_b5cfa7db9d216d26989e2b1ec147ba5f

Frau-Meigs, D. \& Torrent, J. (éds) (2009). Mapping media education policies in the world. Visions, programmes and challenges. New York, NY : The United NationsAlliance of Civilizations \& Grupo Comunicar.

Hartley, J. (2010). The Uses of digital literacy. New Brunswick, NJ : Transaction Publishers.

Hobbs, R. (1996). Teaching media literacy - Yo! Are you hip to this ? Dans D. E. Everette \& E. C. Pease, (éds), Children and the media (pp. 104). New Brunswick, NJ : Transaction Publishers.

Hobbs, R. (1998). The Seven great debates in the media literacy movement. Journal of Communication, 48(1), 16-32.

Hobbs, R. (2011). The state of media literacy: A response to Potter. Journal of Broadcasting \& Electronic Media, 55 (3), 419-430.

Hoechsmann, M. \& Poyntz, S. R. (2012). Media: A Critical introduction. Hichester, Angleterre; Malden, MA : Wiley-Blackwell.

Inchauspé, Paul (éds.). (1997). Réaffirmer l'école. Québec, QC : Ministère de l’Éducation, du Loisir et du Sport.

Jenkins, H. et al. (2009). Confronting the challenges of participatory culture: Media education for the 21st century. Cambridge, MA : MIT Press.

Kumar, K. J. (1992). Une nouvelle définition des objectifs : réflexions venues de l'Inde. Dans L'éducation aux médias dans le monde : nouvelles orientations. Londres, Angleterre : British Film Institute; Paris, France : CLEMI : UNESCO.

Landry, N., Basque, J., \& Agbobli, C. (2015). Éducation aux médias au Canada : état des savoirs et perspective de recherche en communication. Dans A. Kiyindou (éd), Éducation aux médias. Nouveaux enjeux, rôles et statuts des acteurs. Paris, France : L'Harmattan. 
Lewis, J. \& Jhally, S. (1998). The struggle over media literacy. Journal of Communication, 48(1), 109-120.

Livingstone, S. (2004). Media literacy and the challenge of new information and communication technologies. Communication Review, 1(7), 3-14.

Macedo, D. \& Steinberg, S. R. (2007). Media literacy: A reader. New York, NY : Peter Lang.

Marsolais, A. Desaulniers, A., \& Mainville, S. (2007). Soutenir l'appropriation des compétences transversales et des domaines généraux de formation. Québec, QC : ministère de l'Éducation, du Loisir et du Sport.

Martens, H. (2010). Evaluating media literacy education: Concepts, theories and future directions. Journal of Media Literacy Education, 2(1), 1 - 22.

Masterman, L. (1985). Teaching the media. London, Angleterre; New York, NY : Routledge.

Mellouki, M. (éd.) (2010), Promesses et ratés de la réforme de l'éducation au Québec. Québec, QC : Presses de l'Université Laval.

MELS (Ministère de l'Éducation, du Loisir et du Sport). (2006a). Programme de formation de l'école québécoise : éducation préscolaire, enseignement primaire. Québec, QC : gouvernement du Québec, ministère de l'Éducation.

MELS (Ministère de l'Éducation, du Loisir et du Sport). (2006b). Programme de formation de l'école québécoise : enseignement secondaire. premier cycle. Québec, QC : gouvernement du Québec, ministère de l'Éducation, du Loisir et du Sport.

MELS (Ministère de l'Éducation, du Loisir et du Sport). (2007). Programme de formation de l'école québécoise : enseignement secondaire, deuxième cycle. Québec, QC : gouvernement du Québec, ministère de l'Éducation, du Loisir et du Sport.

Ministère de l'Éducation de l'Ontario. (1989). La compétence médiatique : cycles intermédiaire et supérieur. Toronto, ON : ministère de l'Éducation.

Pichette, M. (1999). L'éducation aux médias dans le programme d'études québécois. Chicoutimi, QC : Université du Québec à Chicoutimi.

Piette, J. (1996). Éducation aux médias et fonction critique. Paris, France; Montréal, QC : L'Harmattan. 
Potter, J. W. (2013). Media literacy (7e éd.). Santa Barbara, CA : University of California. Pungente, J., Duncan, B., \& Andersen, N. (2005). The Canadian experience: Leading the way. Yearbook of the National Society for the Study of Education, 104(1), 140-160.

Rossi, J.-P. (2009). Étude de cas sur la représentation des médias dans le matériel pédagogique en éducation aux médias destiné aux enfants du second cycle du primaire (Mémoire de maîtrise inédit). UQAM.

Scheibe, C. \& Rogow, F. (2012). The Teacher's guide to media literacy: Critical thinking in a multimedia world. Thousand Oaks, CA : Corwin.

Silver, A. (2009). Forewords: A European approach to media literacy: Moving toward an inclusive knowledge society. Dans D. Frau-Meigs \& J. Torrent (éd), Mapping media education policies in the world. Visions, programmes and challenges (pp. 11-13 ). New York, NY : The United Nations-Alliance of Civilizations \& Grupo Comunicar.

Table de pilotage du renouveau pédagogique. (2006). Bilan de l'application $d u$ Programme de formation de l'école québécoise - Enseignement primaire, Québec, QC : gouvernement du Québec, ministère de l'Éducation, du Loisir et du Sport.

Wilson, C. \& Duncan, B. (2009). Implementing mandates in media education: The Ontario experience. Dans D. Frau-Meigs \& J. Torrent (éds.), Mapping media education policies in the world. Visions, programmes and challenges (pp. 129-140). New York, NY : The United Nations-Alliance of Civilizations \& Grupo Comunicar.

Yousman, B. (2008). Media literacy: Creating better citizens or better consumers? Dans R. Andersen \& J. Gray (éds), Battleground: The media (pp. 238-247). Westport, CT : Greenwood Press. 